\title{
SOME GROWTH FUNCTIONS OF CONTEXT-DEPENDENT L-SYSTEMS
}

\author{
J. KARHUMÄKI \\ Department of Mathematics, University of Turku, Finland
}

\section{Introduction}

Lindenmayer systems or shortly L-systems were introduced by Lindenmayer, [59] and [60], for describing the development of filamentous organisms. Because of this biological origin one interesting aspect in the study of deterministic L-systems is the theory of growth functions. It is know, [75] and [104], that if a DOL-system grows faster than any polynomial it grows exponentially. In [50] there is an example which shows that the same does not hold for D2L-systems. Here we deal with this example shortly.

Functions $f(n)=k^{n}$ and $g(n)=n^{k}$, where $k$ is a natural number, are DoL growth functions. The inverse functions of these, i.e., logarithm functions and fractional powers, are D2L growth functions, of. [104], but they are not DOL growth functions. So after we have established the existence of a D2L-system with the growth type $2 \frac{1}{2}$, a natural question arises: is there any context-dependent DL-system such that its growth function lies between logarithm functions and fractional powers? In Section 4 of this paper we shall give a positive answer to this question.

For more backround material concerning growth functions the reader is referred to $[75],[104]$ and $[116]$. 


\section{Preliminaries}

We use standard formal language notations, cf. [102]. Here we define only growth types of deterministic L-systems. We say that the growth in a DL-system with the growth function $f(n)$ is exponential or type 3 iff there exist $n_{0}$ and a constant $t>1$ such that

$$
f(n) \geq t^{n} \quad \text { for } n \geq n_{0} \text {. }
$$

Iff there are polynomials $p_{1}(n)$ and $p_{2}(n)$ (with positive rational coefficients) such that $f(n)$ satisfies the condition

$$
p_{1}(n) \leq f(n) \leq p_{2}(n)
$$

we say that the growth is polynomial or type 2. In the case, where the empty word does not belong to the sequence and the growth function is bounded by a constant, we say that the growth is of type 1. Iff the growth function becomes ultimately 0 we say that the growth is of type 0 .

This classification is exhaustive for DoL-systems, cf. [104]. The same does not hold for context-dependent DL-systems. As we mentioned, logarithm functions and fractional powers, which lie between growth types 1 and 2, are D2L growth functions. We say that this kind of

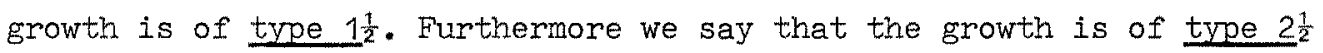
iff the growth function is neither bounded by a polynomial nor of type 3 .

\section{Growth type $2 \frac{1}{2}$}

In this section we give an example of a PD2L-system with the growth type $2 \frac{1}{2}$. First we informally describe the development of our organism.

At certain intervals our organism is of the form $\left(\mathrm{ga}^{\mathrm{k}}\right)^{\mathrm{m}} \mathrm{g}$. Thus, consider the word $\mathrm{ga}^{\mathrm{k}} \mathrm{g}$. The letter $\mathrm{g}$ is called a node. These nodes always send messengers $b$ and $b$ to the right and to the left, respectively. 
At the same time $g$ changes to an inactive form $\bar{g}$ (which does not send any messengers). While moving on, messengers $b$ and $\bar{b}$ duplicate every letter. When $b$ and 5 meet, they create a new node which is in the inactive form. Furthermore, $b$ and 5 disappear and new messengers $f$ and $\vec{i}$ are born. They travel to the right and to the left, respectively. At the beginning, $g$ sends to the right also another messenger (letters $c$ and d). This messenger travels at the rate which is only half of the rates of the other messengers. When this messenger and meet, this slow messenger changes to the messenger f. Now we have three messengers travelling on. Moreover, these are synchronized in the sense that they reach each an inactive node simultaneously. Then this happens, they disappear and transform the nodes to the active form $\mathrm{g}$.

During this process, we increase the number of a's between letters $g$ by one. So the word $g a^{k} g$ has changed to the form $\mathrm{ga}^{k+1} \mathrm{ga}^{\mathrm{k}+1} \mathrm{~g}$, i.e., it has essentially duplicated. Note that the time in which the organism duplicates its length increases linearly.

The development, described above, can be obtained as follows. Consider a PD2L-system with the following productions:

$$
\begin{aligned}
& \mathrm{a}_{\mathrm{a}} \mathrm{g} \rightarrow \mathrm{ba}, \\
& \mathrm{g}_{\mathrm{a}} \mathrm{a} \rightarrow \mathrm{ab} \text {, } \\
& \mathrm{b}^{\mathrm{a}} \rightarrow \mathrm{ab} \text {, } \\
& a_{a} \bar{b} \rightarrow \bar{b} a \text {, } \\
& \mathrm{b}_{\mathrm{a}} \overline{\mathrm{b}} \rightarrow \mathrm{x} \overline{\mathrm{h} f} \text {, } \\
& \mathrm{d}_{\mathrm{a}} \mathrm{x} \rightarrow \mathrm{c} \text { if } \mathrm{x}=\mathrm{a} \text { or } \mathrm{x}=\mathrm{b} \text {, } \\
& f_{a} x \rightarrow f \text { for all } x \text {, } \\
& x_{a} \bar{f} \rightarrow \text { for all } x \text {, } \\
& a_{a}^{e} \rightarrow ? \text {, } \\
& e_{a} a \rightarrow f \text {, } \\
& \bar{\sigma}_{\mathrm{b}} \mathrm{a} \rightarrow \mathrm{d} \text {. } \\
& a_{b^{2}} \rightarrow a \text {, } \\
& a_{b} \bar{b} \bar{x} \bar{g}
\end{aligned}
$$




$$
\begin{aligned}
& a b^{h} \rightarrow a, \\
& a_{b}{ }^{a} \rightarrow a \text {, } \\
& b_{b} a f \text {, } \\
& a_{c} \bar{f} e \text {, } \\
& { }^{x} c^{y} \rightarrow d \text { if } x \neq a \text { or } y \neq \hat{f} \text {, } \\
& a_{d^{x}} \rightarrow \bar{f} \text {, } \\
& x_{d} y \rightarrow a \text { if } x \neq a \text { or } y \neq x \text {, } \\
& \mathrm{x}_{e} \mathrm{y} \rightarrow \mathrm{a} \text { for all } \mathrm{x} \text { and } \mathrm{y} \text {, } \\
& x_{f} y \rightarrow a \text { for all } x \text { and } y \text {, } \\
& d x^{a} \rightarrow f, \\
& \mathrm{x}^{\mathrm{y}} \mathrm{y} \rightarrow \mathrm{a} \text { if } \mathrm{x} \neq \mathrm{d} \text { or } \mathrm{y} \neq \mathrm{a} \text {, } \\
& \alpha_{\mathrm{g}}^{\mathrm{a}} \rightarrow \overline{\mathrm{g}} \mathrm{d}, \\
& \mathrm{a}^{\alpha} \rightarrow \mathrm{a \overline {g }} \text {, } \\
& \mathrm{a}_{\mathrm{g}}^{\mathrm{a}} \rightarrow \mathrm{a} \overline{\mathrm{g}} \mathrm{d}, \\
& \mathrm{x} \overline{\mathrm{g}}^{\mathrm{f}} \rightarrow \mathrm{h} \text { for all } \mathrm{x} \text {, } \\
& f^{-} \mathrm{x} \rightarrow \mathrm{h} \text { for all } \mathrm{x} \text {, } \\
& \alpha^{2}{ }^{a} \rightarrow \overline{h b} \text {, } \\
& a_{h}^{\alpha} \rightarrow \bar{b}, \\
& a_{h}{ }^{a} \rightarrow \overline{b h} b \text {, } \\
& x_{h^{I}} \rightarrow g \text { for all } x \text {, } \\
& f_{h^{x}} \rightarrow g \text { for all } x \text {, } \\
& \mathrm{y}_{\mathrm{x}^{2}}{ }^{\mathrm{z}} \mathrm{x} \quad \text { otherwise. }
\end{aligned}
$$

Above $\alpha$ is the input from the environment. To simplify the growth function we use two kinds of nodes, $g$ and $h$. The details of the following are in [50]. If we take $\mathrm{ga}^{4} \mathrm{~g}$ to the 19 th word of our system, then after $3 n^{2}$ steps, where $n \geq 3$, the organism is of the form

$$
\left(h a^{2 n-1}\right)^{24^{n-3}} n
$$

So the growth function $f(n)$ satisfies the condition

$$
f\left(3 n^{2}\right)=2 n 2^{2 n-5}+1, n \geq 3 .
$$

From this we obtain easily, because our system is $\lambda$-free, the result 
that there exists $n_{0}$ such that

$$
2^{\sqrt{n}} \leq f(n) \leq\left(2^{\sqrt{3}}\right)^{\sqrt{n}} \quad, n \geq n_{0} .
$$

So our system is indeed of the growth type $2 \frac{1}{2}$.

\section{Growth between logarithm functions and fractional powers}

In this section we show, how we can obtain PD1L growth functions which lie between logarithm functions and fractional powers. Particularly, we show that there is a PD1L-system such that its growth function is asymptotically equal to the function $\left({ }^{2} \log n\right)^{2}$. The development of our organism can be described as follows.

The organism consists of two parts. The left one is called the growing part and the right one is called the control part. The growing part is a DL-system, and it determines essentially the length of the organism. The purpose of the control part is to tell to the growing part when it has to take one step. This can be accomplished by means of a messenger which is sent at certain intervals by the control part and which travels through the growing part.

In the next example the control part will be a PD1L-system with the growth function asymptotically equal to the function ${ }^{2} \log n$. Furthermore, the growing part will be a PDOL-system which grows asymptotically like the function $n^{2}$. So it is needed $2^{n}$ steps to change the length of the organism from $n^{2}$ to $(n+1)^{2}$.

Now we go to the formal example. Let $\mathrm{H}$ be the following PDOLsystem

$$
H=\left(\{a, b, c\}, v_{1}=c c,\{a \rightarrow a, b \rightarrow b a, c \rightarrow c b a\}\right) .
$$

(Note that we have not specified the axiom $v_{0}$ ). Using the methods of [98], one can easily see that the growth function $g(n)$ of this system satisfies the equation

$$
g(n)=(n+1)^{2}-(n+1) \quad, n \geq 1 .
$$

This will be the growing part of our system. 
Now we define the whole system $G$. The alphabet $V$ is

$$
V=\{e, 0,1, s\} \cup V_{1} \cup \bar{V}_{1}
$$

where $V_{1}=\{a, b, c\}$ and $\bar{V}_{1}=\{\bar{a}, \bar{b}, \bar{c}, \bar{e}\}$. The input from the environment is $g$. The axiom is not specified, but $w_{3}$ is the word cceo. The productions are as follows:

$$
\begin{array}{ll}
a^{-x} \rightarrow a & \text { for } a l l x \in V, \\
b^{x} \rightarrow b a & \text { for all } x \in V, \\
\bar{c}^{-x} \rightarrow c b a & \text { for all } x \in V, \\
e^{-x} \rightarrow e \quad \text { for all } x \in V, \\
e^{S} \rightarrow \bar{e} 0, \\
x^{\bar{y}} \rightarrow \bar{x}, \quad \text { for all } x \in V_{1} \text { and } \bar{y} \in \bar{V}_{1}, \\
0^{g} \rightarrow 1, \\
0^{S} \rightarrow 1, \\
1^{g} \rightarrow S, \\
1^{S} \rightarrow S, \\
S^{8} \rightarrow 1, \\
S^{0} \rightarrow 0, \\
S^{1} \rightarrow 0, \\
x^{y} \rightarrow x, \quad
\end{array}
$$

Assertion 1. Let $\mathrm{P}$ be an arbitrary word over $\mathrm{V}$. Then for all

$i \geq 1$, the following two conditions hold
(i) $\quad \mathrm{PO}^{i} \Rightarrow^{2^{i}} \mathrm{p}^{\prime} 1^{i+1}$ and
(ii) if $P O 1^{i} \Rightarrow^{k} P^{\prime} X Q$, where $k<2^{i}, X \in V$ and $\lg (Q)=i$, then $X$ differs from the letter $S$.

Proof. Because the productions for the letters $s, 0$ and 1 are length preserving, the word $P$ above derives exactly the word $P^{\text {' in }}$ both cases. Moreover, the rewriting of our system depends only on the right neighbour of a letter. Thus, we may assume that $P$ and $P^{\prime}$ are empty words.

Consider the derivations 


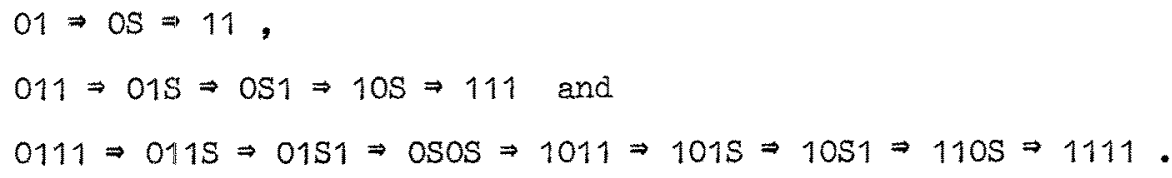

So the Assertion is true if $i \leq 3$.

Assume now that the Assertion is true for every $k \leq i-1$, where $i>3$. Consider now the derivation starting from the word $01^{i}$. Let $j$ be the least natural number such that $i+1 \leq 2^{j}$. Then clearly $3 \leq j \leq i-1$. After $i+1$ steps the word $01^{i}$ has changed to the form $10 \mathrm{R}$. So by the induction hypothesis, the considered derivation begins as follows:

$$
\begin{aligned}
& 01^{i-4} 1111 \\
& 01^{i-4} 111 \mathrm{~s} \\
& 01^{i-4} 11 \mathrm{~s} 1 \\
& 01^{i-4} 1 \mathrm{SOS} \\
& 01^{i-4} \text { S011 } \\
& -\ldots+2^{j-1} \text { steps } \\
& 10^{i-j} j^{j} \\
& -\cdots+2^{i-2} \text { steps } \\
& 101^{i-1}
\end{aligned}
$$

Note that during this part of the derivation the leftmost symbol differs from $S$. During the next $2^{i-1}$ steps the second letter from the left differs from $S$ by induction hypothesis. So the leftmost symbol differs from 1. Furthermore, the word $01^{i-1}$ changes to the word $1^{i}$.

Thus, the word $01^{i}$ has changed in

$$
1+1+2+\cdots+2^{i-1}=2^{i}
$$

steps to the word $1^{i+1}$, and in every stage the leftmost symbol differs from 5 . So we have proved Assertion 1.

Assertion 2. For all $i \geq 1$, the following is true

$$
e 1^{i}=^{2^{i+1}} \quad e^{i+1} \text { and }
$$

(ii) if $e 1^{i} \Rightarrow E Q$, where $k<2^{i+1}$ and $E \in\{e, \bar{e}\}$, then $E=\bar{e}$ 
if and only if $k=i+1$.

Proof. One can easily see that the Assertion is true if $i \leq 3$. If $i>3$, the derivation starting from the word $e 1^{i}$ begins as follows.

$$
e 1^{i-4} 1111 \Rightarrow e 1^{i-4} 111 S \Rightarrow e 1^{i-4} 11 S 1 \Rightarrow e 1^{i-4} 1 \text { sos } \Rightarrow e 1^{i-4} \text { so11. }
$$

Now $S$ is travelling on to the left. At each step it changes to 0 and the next 1 changes to $S$. When $S$ meets $e$, it disappears and e changes to the word $\bar{e}$. At the next step this bar disappears.

By Assertion 1, the $(i+1)$ st letter from the right differs from $S$ during the first

$$
1+1+2+\ldots+2^{i}=2^{i+1}
$$

steps. Thus, by Assertion 1, the word $e 1^{i}$ changes in $2^{i+1}$ steps to the word $e 1^{i+1}$. Clearly, the claim (ii) is also true.

Let $E(H)$ and $E(G)$ be the sequences generated by $H$ and $G$, respectively. Denote

$$
\begin{aligned}
& E(H)=v_{0}, v_{1}=c c, v_{2}, \ldots \\
& E(G)=w_{0}, w_{1}, w_{2}, w_{3}=c c e 0, w_{4}, \ldots .
\end{aligned}
$$

The whole derivation according to $G$ is as follows:

$$
\begin{aligned}
& w_{3}=\quad \operatorname{cce} 0 \\
& w_{4}=\quad \operatorname{cce} 1 \\
& \operatorname{coes} \\
& \text { ccēo1 } \\
& \text { c̄ēeos } \\
& \mathrm{w}_{8}=\quad \overline{\mathrm{ccbae}} 11 \\
& \text { cbacbae1s } \\
& \text { cbacbaes1 } \\
& \text { cbacbaēoos } \\
& \text { cbacbāe011 } \\
& \text { cbac5ae015 } \\
& \text { cbac̄baaeos1 }
\end{aligned}
$$




$$
\begin{aligned}
& \text { cbācbabaae10S } \\
& w_{16}=\text { cbacbabaae } 111 \\
& \text { çbaacbabaae11s } \\
& v_{3} e^{1 S 1} \\
& w_{32}=\ldots \text { e1111 } \\
& v_{4} e 111 S \\
& w_{64}=\quad v_{5} e^{e 11111} \\
& w_{2^{i+1}}=\quad v_{i} e^{i}
\end{aligned}
$$

The word $v_{i-1} e 1^{i-1}$ changes in $(i+1)+1 g\left(v_{i-1}\right)$ steps to the word $v_{i} E P$, where $E \in\{e, \bar{e}\}$. Thus, it follows from the fact

$$
2^{i} \geq(i+1)+\left(i^{2}-i\right) \quad, i \geq 5
$$

that the general formula for $w_{2} i+1$ is indeed as above.

If $f(n)$ is the growth function of our system, then

$$
f\left(2^{i+1}\right)=(i+1)^{2}, i \geq 5 \text {. }
$$

Assume $n \in\left[2^{i+1}, 2^{i+2}\right]$. Then, because our system is $\lambda$-free, the following approximations are true for $n \geq 32$

$$
\begin{aligned}
& f(n) \leq f\left(2^{i+2}\right)=(i+2)^{2} \leq\left({ }^{2} \log (n)+1\right)^{2} \text { and } \\
& f(n) \geq f\left(2^{i+1}\right)=(i+1)^{2} \geq\left({ }^{2} \log (n)-1\right)^{2} .
\end{aligned}
$$

Thus, the function $f(n)$ is asymptotically equal to the function $\left({ }^{2} \log n\right)^{2}$.

We can generalize the above example as follows. Let $p$ and $r$ be natural numbers. The function $(n+1)^{r}-(n+1)$ is a PDOL growth function, cf. [111]. Moreover, there exists a PD1L-system with the growth function asymptotically equal to the function $p_{\log } \mathrm{n}$ (P. Vitányi, personal communication). So the above construction gives us a PD1L-system with the growth function asymptotically equal to the function $\left({ }^{p_{l o g}} n\right)^{r}$. 